\title{
CONCENTRAÇÕES DE CLORETO DE CÁLCIO E TEMPOS DE ARMAZENAMENTO NOS TEORES DE ACUÚCARES REDUTORES DE UVAS CV RED GLOBE (Vitis vinifera $\mathrm{L}$ )
}

\author{
Calcium chloride concentrations and storage time on reducing sugar contents \\ of grape cv red globe (Vitis vinifera $\mathrm{L}$ )
}

\author{
Geny Lopes de Carvalho ${ }^{1}$, Luiz Carlos de Oliveira Lima² José Daniel Silva $^{3}$, \\ Heloísa Helena Siqueira ${ }^{4}$, Elisa Carvalho Morais ${ }^{3}$
}

\begin{abstract}
RESUMO
Objetivou-se avaliar a ação do cloreto de cálcio na doçura pós-colheita de uvas 'Red Globe' (Vitis vinifera L) cultivadas em Jales, noroeste do Estado de São Paulo, foram adotados os procedimentos: os cachos foram tratados por imersão durante 10 minutos em soluções $\mathrm{CaCl}_{2}$ 0, 1, 2 e $3 \%$ e deixados por 30, 60 e 90 dias a uma temperatura de $0^{\circ} \mathrm{C}$ a $3^{\circ} \mathrm{C}, 90 \pm 3 \%$ de UR e $0,2-0,3 \mathrm{~m} / \mathrm{s}$ de velocidade do ar, após os quais foram deixados em temperatura ambiente $\left(20\right.$ a $\left.25^{\circ} \mathrm{C}\right)$, para avaliações aos 0,3 e 6 dias. O delineamento experimental foi inteiramente casualizado em esquema fatorial $4 \times 3 \times 3$ (concentrações de $\mathrm{CaCl}_{2} \times$ tempo de armazenamento refrigerado $\mathrm{x}$ tempo de armazenamento em temperatura ambiente) com três repetições. Foram feitas avaliações de açúcares redutores

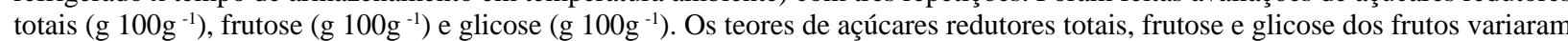
com a aplicação de $\mathrm{CaCl}_{2}$, tempos de armazenamento a frio e em temperatura ambiente. Destaca-se a frutose cujos teores em todas as concentrações de $\mathrm{CaCl}_{2}(1,2$ e 3\%), aos 30 e 60 dias de armazenamento refrigerado e 3 e 6 dias em temperatura ambiente, foram superiores aos teores dos frutos controle. Conclui-se que tratamentos com $\mathrm{CaCl}_{2}$ propicia às uvas melhor sabor, sendo indicada a concentração de $\mathrm{CaCl}_{2} 2 \%$ e o armazenamento a frio por 60 dias e em temperatura ambiente até seis dias.
\end{abstract}

Termos para indexação: Uva de mesa, doçura, Pós-colheita, Vitis vinifera L.

\section{ABSTRACT}

This work was carried out with the aim of evaluating the calcium chloride influence on both post harvest sweetness of grapes 'Red Globe' (Vitis vinifera L) grown in Jales, in the northwest of São Paulo State. The procedures adopted were as follows: banches were treated in a $\mathrm{CaCla}_{2} 1 \%, 2 \%$, and $3 \%$ immersion for 10 minutes and then left at na $0^{\circ} \mathrm{C}$ tempereture, $90 \pm 3 \%$ relative humidity, and $0,2-0,3 \mathrm{~m} / \mathrm{s}$ air speed for 30,60 , and 90 days after which they were left at room temperature $\left(20\right.$ to $\left.25^{\circ} \mathrm{C}\right)$ for evaluation. A ramdomized block design with a $4 \times 3 \times 3\left(\mathrm{CaCl}_{2}\right.$ concentration $\mathrm{x}$ cold storage $\mathrm{x}$ room storage $)$ factorial arrangement and three

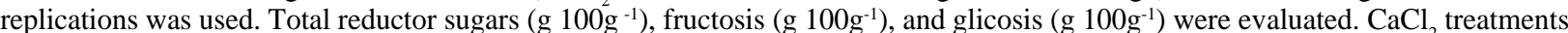
as well as storage time at both cold and room temperature were found to cause total reductor sugars, fructosis, and glicosis content in the fruits to vary/differ/be at variance degree. Fructosis (outstanding) contents were found to be higher than those in the control fruits in all $\mathrm{CaCl}_{2}$ concentrations after both 30 and 60 storage days at cold temperature, and 3 and 6 days at room temperature. Conclusion can be made that $\mathrm{CaCl}_{2}$ treatments promote better flavour to grapes. The $\mathrm{CaCl}_{2} 2 \%$ concentration, the cold storage for 60 days, and the room storage for up to 6 days should be used.

Index terms: Grape of table, sweetness, Post harvest, Vitis vinifera $\mathrm{L}$.

(Recebido em 29 de março de 2006 e aprovado em 22 de maio de 2007)

\section{INTRODUÇÃO}

O sabor é um importante atributo de qualidade. O conteúdo e a composição dos açúcares têm papel fundamental no sabor, sendo também indicadores de estádio de maturação dos frutos (KADER, 1999; WANG,
1999). Em uvas, os principais açúcares são a frutose e a glicose, podendo a sacarose estar presente em algumas cultivares, porém em baixas concentrações.

Vários fatores influenciam os porcentuais de açúcares das uvas e, entre eles, são ressaltadas as características genéticas de cultivares, as condições

\footnotetext{
${ }^{1}$ Mestre em Ciências dos Alimentos - Departamento de Ciência dos Alimentos/DCA - Universidade Federal de Lavras/UFLA - Cx. P. 3037 - $37200-000$ Lavras, MG - genylopes13@yahoo.com.br

2Doutor em Ciência dos Alimentos, Professor Adjunto - Departamento de Ciência dos Alimentos/DCA - Universidade Federal de Lavras/UFLA - Cx. P. 3037 - 37200-000 - Lavras, MG - Icolima@ufla.br

${ }^{3}$ Graduados em Engenharia de Alimentos - Departamento de Ciência dos Alimentos/DCA - Universidade Federal de Lavras/UFLA - Cx. P. 3037 - $37200-000$ Lavras, MG - jdalimentos@hotmail.com; elisa_ufla@hotmail.com

${ }^{4}$ Doutoranda em Ciência dos Alimentos - Departamento de Ciência dos Alimentos/DCA - Universidade Federal de Lavras/UFLA - Cx. P. 3037 - $37200-000$ -

Lavras, MG - eloísa.elias@yahoo.com.br
} 
climáticas, o tipo de solo onde são cultivadas as videiras, etc (PEREIRA, 2001; PEYNAUD, 1997; RIZZON et al., 1998).

A temperatura tem efeito decisivo na síntese dos açúcares dos frutos. Dessa forma, a insolação desempenha papel importante no incremento de seus teores (NOGUEIRA, 1984). Em regiões tropicais, como o noroeste de São Paulo, onde as chuvas ocorrem durante o período de maturação dos frutos, raramente as uvas atingem teores de sólidos solúveis superiores a $14^{\circ}$ Brix. Dessa forma, há necessidade de utilização de técnicas que venham a melhorar os teores de sólidos solúveis (açúcares) das uvas provenientes dessa região. Em vários frutos, o cálcio vem sendo utilizado visando prolongar a vida de armazenamento e qualidade dos frutos (CENCI, 1994). Além disso, a sua associação com as membranas celulares através de enlace com os fosfolipídeos lhes conferem textura mais firme, dando-lhes maior resistência às injúrias de natureza fisiológica, microbiana e mecânica e, conseqüentemente, redução da permeabilidade com decréscimo no processo respiratório, na produção de etileno e na perda de água, retardando a senescência. A redução da produção de $\mathrm{CO}_{2}$ (atividade respiratória), provoca aumento no teor de açúcares simples (CHITARRA \& CHITARRA, 2005; FRÁGUAS \& SILVA, 1998).

Como o poder adoçante varia de acordo com o tipo de açúcar (frutose, glicose, sacarose), torna-se necessário, para determinar a qualidade das uvas, analisá-los de forma separada. Sendo assim, o objetivo desse trabalho foi o de investigar o efeito da aplicação pós-colheita de diferentes concentrações de cloreto de cálcio, em diferentes tempos de armazenamento refrigerado seguidos de temperatura ambiente, nos açúcares redutores totais, frutose e glicose, de uvas 'Red Globe' cultivadas em Jales, noroeste de São Paulo.

\section{MATERIAL E MÉTODOS}

\section{Material Experimental}

O presente trabalho foi realizado no Laboratório Pós-Colheita de Frutos e Hortaliças do Departamento de Ciência dos Alimentos - UFLA. Foram utilizadas uvas 'Red Globe' (Vitis vinifera L), colhidas em 10-11-2004, provenientes da regional agrícola de Jale, SP (latitude $20^{\circ} \mathrm{S}$, longitude $50^{\circ} \mathrm{W}$, altitude $450-550 \mathrm{~m}$, precipitação anual 1300 mm e temperatura média $22,3^{\circ} \mathrm{C}$ ).

\section{Tratamentos e Delineamento Experimental}

No laboratório, foram selecionados e pesados 144 cachos de uvas, colhidos e embalados em 10-11-2004 e divididos em quatro lotes que foram submetidos aos seguintes tratamentos: controle $\left(\mathrm{CaCl}_{2} 0 \%\right)$; imersão por 10 minutos em solução de $\mathrm{CaCl}_{2} 1 \%$; imersão por 10 minutos em solução $\mathrm{CaCl}_{2} 2 \%$; imersão por 10 minutos em solução $\mathrm{CaCl}_{2} 3 \%$. Após, eles foram secados em temperatura ambiente $\left(20\right.$ a $\left.25^{\circ} \mathrm{C}\right)$.

Trinta e seis (36) cachos foram utilizados para caracterização da qualidade no dia da instalação do experimento. Os demais cachos foram divididos em 12 caixas com 9 cachos cada uma e armazenados por 30, $60 \mathrm{e}$ 90 dias, a uma temperatura de 0 a $3^{\circ} \mathrm{C}, 90 \pm 3 \%$ de UR e $0,2-$ $0,3 \mathrm{~m} / \mathrm{s}$ de velocidade do ar. Cada conjunto de nove cachos foi separado em três subconjuntos com três cachos em cada um, que foram armazenados por 0, 3 e 6 dias em temperatura ambiente 20 a $25^{\circ} \mathrm{C}$, após a retirada da câmara fria. Cada cacho desses subconjuntos constituiu a parcela experimental.

Os tratamentos em estudo foram formados pelas combinações entre as concentrações de $\mathrm{CaCl}_{2}(0,1,2$ e $3 \%)$, os três tempos de armazenamento refrigerado $(30,60$ e 90 dias) e os três tempos em temperatura ambiente $(0,3$ e 6 dias). $\mathrm{O}$ delineamento experimental foi inteiramente casualizado, com três repetições, sendo os tratamentos dispostos em esquema fatorial $4 \times 3 \times 3$ (concentrações de $\mathrm{CaCl}_{2} \mathrm{x}$ tempos de armazenamento refrigerado $\mathrm{x}$ tempos de armazenamento sob temperatura ambiente).

\section{Análise Estatística}

As análises de variância dos resultados obtidos de açúcares redutores totais, frutose e glicose foram realizadas com o auxílio do programa SISVAR (FERREIRA, 2000).

\section{Metodologias Analíticas \\ Açúcares Redutores Totais}

A extração foi feita pelo método de Lane-Enyon, citado pela AOAC (1990), e os açúcares determinados pela técnica de Somogy, adaptada por Nelson (1994).

\section{Frutose}

A quantificação foi feita segundo método de Ribéreau-Gayon \& Reynaud (1964). O método é baseado na coloração azul que a frutose forma em meio ácido, quando reage com a difenilamina. A coloração foi extraída com o álcool amílico e lida no espectrofotômetro a $640 \mathrm{~nm}$.

\section{Glicose}

Obtida pela diferença entre teores de açúcares redutores totais, segundo técnica de Somogy, adaptada por Nelson (1994) e frutose. 


\section{RESULTADOS E DISCUSSÃO}

Ao 0 dia de armazenamento em temperatura ambiente e 30 dias de armazenamento refrigerado, as uvas tratadas com $\mathrm{CaCl}_{2} 2 \%$ apresentaram maiores teores de açúcares redutores que os teores dos frutos-controle (Tabela 1). Ao 0 dia de armazenamento, em temperatura ambiente e 60 dias de armazenamento refrigerado, destacaram-se os frutos tratados com $\mathrm{CaCl}_{2} 1$ e $3 \%$, enquanto que ao 0 dia de armazenamento em temperatura ambiente e 90 dias de armazenamento refrigerado sobressaíram os frutos tratados com $\mathrm{CaCl}_{2} 1 \%$ e controle, com maiores teores de açúcares redutores.

Aos 3 dias de armazenamento em temperatura ambiente e aos 60 e 90 dias de armazenamento refrigerado, as uvas tratadas com $\mathrm{CaCl}_{2} 2 \%$ apresentaram teores elevados de açúcares redutores totais, ao passo que aos 3 dias de prateleira e aos 30 dias de armazenamento, as uvas tratadas com a mesma concentração de $\mathrm{CaCl}_{2} 2 \%$ tiveram teores de açúcares redutores iguais aos do controle, porém superiores aos teores dos frutos tratados com $\mathrm{CaCl}_{2} 1$ e $3 \%$.

Em 6 dias de armazenamento em temperatura ambiente e 30 dias de armazenamento, os frutos-controle e os de tratamento $\mathrm{CaCl}_{2}, 3 \%$ tiveram teores de açúcares redutores totais superiores aos dos frutos tratados com $\mathrm{CaCl}_{2} 1$ e $2 \%$, enquanto que aos 6 dias de armazenamento em temperatura ambiente e 60 dias de armazenamento, os maiores teores de açúcares redutores totais ocorreram no tratamento $\mathrm{CaCl}_{2} 3 \%$. Aos 6 dias de armazenamento em temperatura ambiente e 90 dias de armazenamento $\mathrm{CaCl}_{2}$ $1 \%$,os teores de açúcares redutores foram iguais aos dos frutos-controle e ambos superiores aos teores das uvas tratadas com $\mathrm{CaCl}_{2} 2$ e $3 \%$. Essa inconstância em relação aos efeitos das concentrações de cálcio pode ter ocorrido em razão do processo de senescência que ocorreu após os 90 dias de armazenamento, nas concentrações de cloreto de cálcio 2 e $3 \%$. As altas quantidades de açúcares podem ser indicativo de uma maior taxa de conversão dos ácidos orgânicos em açúcares e do aumento na hidrólise de polímeros estruturais da parede celular (BRODY et al., citados por CAMPOS et al., 2004).

Os teores de frutose (Tabela 2) ao 0 dia de armazenamento, em temperatura ambiente e aos 30 dias de armazenamento refrigerado, nos frutos tratados com $\mathrm{CaCl}_{2}$ $1 \%$ foram inferiores aos do controle $\left(\mathrm{CaCl}_{2} 0 \%\right)$. Nas demais concentrações, os teores de frutose foram de 7,94g $100 \mathrm{~g}^{-1}$

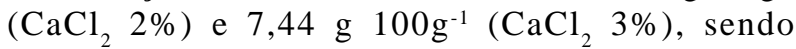

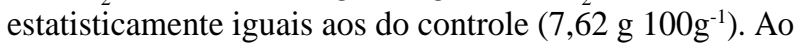
0 dia de armazenamento em temperatura ambiente e aos 60 dias de armazenamento refrigerado, apenas os frutos tratados com $\mathrm{CaCl}_{2} 2 \%$ apresentaram teores de frutose iguais aos do controle, enquanto que aos 90 dias de armazenamento refrigerado, os frutos com maiores teores de frutose, foram os do controle.

Tabela 1 - Teores médios de açúcares redutores $\left(\mathrm{g} 100 \mathrm{~g}^{-1}\right)$ em uvas 'Red Globe', tratadas com $\mathrm{CaCl}_{2}(0,1,2$ e $3 \%)$ e armazenadas em temperatura ambiente por 0, 3 e dias e temperatura refrigerada por 30, 60 e 90 dias.

\begin{tabular}{|c|c|c|c|c|c|c|c|c|c|}
\hline \multirow{4}{*}{$\begin{array}{l}\text { Concentração } \\
\text { de } \mathrm{CaCl}_{2}(\%)\end{array}$} & \multicolumn{9}{|c|}{ Tempo de armazenamento sob temperatura ambiente (dias) } \\
\hline & \multicolumn{3}{|c|}{0} & \multicolumn{3}{|c|}{3} & \multicolumn{3}{|c|}{6} \\
\hline & \multicolumn{9}{|c|}{ Tempo de armazenamento refrigerado (dias) } \\
\hline & 30 & 60 & 90 & 30 & 60 & 90 & 30 & 60 & 90 \\
\hline 0 & $\begin{array}{l}9,95 \\
(\mathrm{aA})\end{array}$ & $\begin{array}{c}10,03 \\
(\mathrm{bA})\end{array}$ & $\begin{array}{l}10,75 \\
(\mathrm{cB})\end{array}$ & $\begin{array}{c}10,30 \\
(\mathrm{cA})\end{array}$ & $\begin{array}{l}10,17 \\
(\mathrm{bA})\end{array}$ & $\begin{array}{l}11,23 \\
(\mathrm{cB})\end{array}$ & $\begin{array}{l}11,05 \\
(\mathrm{cC})\end{array}$ & $\begin{array}{c}9,28 \\
(\mathrm{bA})\end{array}$ & $\begin{array}{c}10,45 \\
(\mathrm{cB})\end{array}$ \\
\hline 1 & $\begin{array}{l}9,97 \\
(\mathrm{aA})\end{array}$ & $\begin{array}{l}10,88 \\
(\mathrm{~dB})\end{array}$ & $\begin{array}{c}11,17 \\
(\mathrm{dC})\end{array}$ & $\begin{array}{l}8,86 \\
(\mathrm{aA})\end{array}$ & $\begin{array}{l}9,94 \\
(\mathrm{bC})\end{array}$ & $\begin{array}{l}9,36 \\
(\mathrm{aB})\end{array}$ & $\begin{array}{l}9,03 \\
(\mathrm{aA})\end{array}$ & $\begin{array}{l}9,10 \\
(\mathrm{bA})\end{array}$ & $\begin{array}{l}9,99 \\
(\mathrm{cB})\end{array}$ \\
\hline 2 & $\begin{array}{c}11,24 \\
(\mathrm{bC})\end{array}$ & $\begin{array}{l}8,53 \\
(\mathrm{aA})\end{array}$ & $\begin{array}{l}9,58 \\
(\mathrm{bB})\end{array}$ & $\begin{array}{c}10,30 \\
(\mathrm{cA})\end{array}$ & $\begin{array}{l}11,36 \\
(\mathrm{cB})\end{array}$ & $\begin{array}{c}11,59 \\
(\mathrm{~dB})\end{array}$ & $\begin{array}{c}10,03 \\
(\mathrm{bC})\end{array}$ & $\begin{array}{l}8,48 \\
(\mathrm{aA})\end{array}$ & $\begin{array}{l}9,61 \\
\text { (bB) }\end{array}$ \\
\hline 3 & $\begin{array}{l}9,97 \\
(\mathrm{aB}) \\
\end{array}$ & $\begin{array}{c}10,32 \\
(\mathrm{cC})\end{array}$ & $\begin{array}{c}7,810 \\
(\mathrm{aA})\end{array}$ & $\begin{array}{l}9,82 \\
(\mathrm{bB}) \\
\end{array}$ & $\begin{array}{l}8,92 \\
(\mathrm{aA})\end{array}$ & $\begin{array}{c}10,28 \\
(\mathrm{bC})\end{array}$ & $\begin{array}{c}11,25 \\
(\mathrm{cC})\end{array}$ & $\begin{array}{r}70,87 \\
(\mathrm{cB}) \\
\end{array}$ & $\begin{array}{l}8,01 \\
(\mathrm{aA})\end{array}$ \\
\hline
\end{tabular}

$\mathrm{CV}=1,56$

CV = coeficiente de variação;

Letras maiúsculas - indicam diferenças na horizontal;

Letras minúsculas - indicam diferenças na vertical.

Ciênc. agrotec., Lavras, v. 32, n. 3, p. 894 -899, maio/jun., 2008 


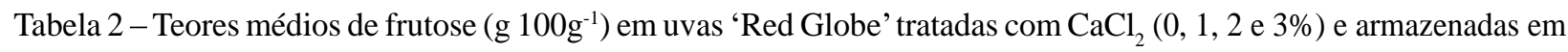
temperatura ambiente por 0,3 e 6 dias e em temperatura refrigerada por 30, 60 e 90 dias.

\begin{tabular}{|c|c|c|c|c|c|c|c|c|c|}
\hline & \multicolumn{9}{|c|}{ Tempo de armazenamento em temperatura ambiente (\%) } \\
\hline & & 0 & & & 3 & & & 6 & \\
\hline \multirow{2}{*}{$\begin{array}{l}\text { Concentração } \\
\text { de } \mathrm{CaCl}_{2}(\%)\end{array}$} & \multicolumn{9}{|c|}{ Tempo de armazenamento refrigerado (\%) } \\
\hline & 30 & 60 & 90 & 30 & 60 & 90 & 30 & 60 & 90 \\
\hline $0 \%$ & $\begin{array}{l}7,62 \\
(\mathrm{bA})\end{array}$ & $\begin{array}{l}7,95 \\
(\mathrm{bA})\end{array}$ & $\begin{array}{l}8,08 \\
(\mathrm{cA})\end{array}$ & $\begin{array}{l}5,93 \\
(\mathrm{aA})\end{array}$ & $\begin{array}{l}6,49 \\
(\mathrm{aB})\end{array}$ & $\begin{array}{l}8,77 \\
(\mathrm{bC})\end{array}$ & $\begin{array}{l}6,34 \\
(\mathrm{aA})\end{array}$ & $\begin{array}{l}6,45 \\
(\mathrm{aA})\end{array}$ & $\begin{array}{l}6,88 \\
\text { (bB) }\end{array}$ \\
\hline $1 \%$ & $\begin{array}{l}6,10 \\
(\mathrm{aA})\end{array}$ & $\begin{array}{l}6,95 \\
(\mathrm{aB})\end{array}$ & $\begin{array}{l}6,94 \\
(\mathrm{bB})\end{array}$ & $\begin{array}{l}7,34 \\
(\mathrm{cB})\end{array}$ & $\begin{array}{l}8,09 \\
(\mathrm{bC})\end{array}$ & $\begin{array}{l}6,31 \\
(\mathrm{aA})\end{array}$ & $\begin{array}{l}7,97 \\
(\mathrm{cB})\end{array}$ & $\begin{array}{l}8,64 \\
(\mathrm{bC})\end{array}$ & $\begin{array}{l}6,28 \\
(\mathrm{aA})\end{array}$ \\
\hline $2 \%$ & $\begin{array}{l}7,94 \\
(\mathrm{bB})\end{array}$ & $\begin{array}{l}8,00 \\
(\mathrm{bB})\end{array}$ & $\begin{array}{l}6,27 \\
(\mathrm{aA})\end{array}$ & $\begin{array}{l}6,62 \\
(\mathrm{bA})\end{array}$ & $\begin{array}{l}7,77 \\
(\mathrm{bB})\end{array}$ & $\begin{array}{l}6,31 \\
(\mathrm{aA})\end{array}$ & $\begin{array}{l}7,01 \\
\text { (bB) }\end{array}$ & $\begin{array}{l}6,85 \\
(\mathrm{aB})\end{array}$ & $\begin{array}{l}6,33 \\
(\mathrm{aA})\end{array}$ \\
\hline $3 \%$ & $\begin{array}{l}7,44 \\
(\mathrm{bB})\end{array}$ & $\begin{array}{l}7,15 \\
(\mathrm{aB})\end{array}$ & $\begin{array}{l}6,37 \\
(\mathrm{aA})\end{array}$ & $\begin{array}{l}6,97 \\
(\mathrm{bB})\end{array}$ & $\begin{array}{l}6,71 \\
(\mathrm{aB})\end{array}$ & $\begin{array}{r}6,13 \\
(\mathrm{aA})\end{array}$ & $\begin{array}{l}7,09 \\
(\mathrm{bB})\end{array}$ & $\begin{array}{l}6,69 \\
(\mathrm{aB})\end{array}$ & $\begin{array}{l}6,26 \\
(\mathrm{aA})\end{array}$ \\
\hline
\end{tabular}

$\mathrm{CV}(\%)=3,89$

$\mathrm{CV}=$ coeficiente de variação;

Letras maiúsculas - indicam diferenças na horizontal;

Letras minúsculas - indicam diferenças na vertical.

Em 3 e 6 dias de armazenamento em temperatura ambiente e aos 30 e 60 dias de armazenamento refrigerado, os tratamentos com $\mathrm{CaCl}_{2}(0,1,2$ e $3 \%)$ propiciaram aos frutos maiores teores de frutose que os do controle, enquanto que, aos 90 dias de armazenamento refrigerado, foram os frutos controle que apresentaram maiores teores de frutose. Com o tempo de armazenamento ocorreram aumentos nos teores de frutose dos 30 para 60 dias seguidos de decréscimo aos 90 dias, em 41,67\% dos tratamentos; decréscimos em $33,37 \%$ e aumento em $25 \%$ dos tratamentos. Sendo a frutose o açúcar de maior poder adoçante, ressalta-se o resultado da aplicação do cálcio na doçura do fruto melhorando sua qualidade. O cálcio limita a difusão dos substratos do vacúolo para o citoplasma diminuindo a respiração dos frutos e conseqüentemente a concentração de frutose 2,6-bifosfato e fluxo glicolítico (BRADY, 1987; CHITARRA \& CHITARRA, 2005).

Com relação aos teores de glicose, observa-se que, ao 0 dia de armazenamento em temperatura ambiente e aos 30 e 90 dias de armazenamento refrigerado, os frutos tratados com $\mathrm{CaCl}_{2} 1$ e $2 \%$ apresentaram teores de glicose superiores aos do controle e que aos 60 dias de armazenamento refrigerado foram os tratamentos com $\mathrm{CaCl}_{2}$ 1 e $3 \%$ que conferiram aos frutos maiores teores de glicose (Tabela 3).
Aos 3 dias de armazenamento em temperatura ambiente, tanto aos 30 quanto aos 60 dias de armazenamento refrigerado, os frutos-controle sobressaíram com maiores teores de glicose. Ressalta-se, porém, que os frutos tratados com $\mathrm{CaCl}_{2} 3 \%$, apesar de apresentarem teores de glicose numericamente inferiores, foram estatisticamente semelhantes aos teores do controle. Aos 90 dias de armazenamento refrigerado em todos os tratamentos com $\mathrm{CaCl}_{2}$ os teores de glicose dos frutos foram superiores aos do controle, sobressaindo o tratamento $\mathrm{CaCl}_{2} 2 \%$ com teores mais elevados $(5,29 \%)$. Aos 6 dias de prateleira, apenas nos frutos armazenados em câmara refrigerada por 60 dias e tratados com $\mathrm{CaCl}_{2} 3 \%$ os teores de glicose foram superiores aos dos frutos-controle.

Com o armazenamento refrigerado ocorreram decréscimos nos teores de glicose dos 30 para os 60 dias, seguido de aumento dos 60 para os 90 dias em $50 \%$ dos tratamentos. Em 16,67\% dos tratamentos ocorreram aumento dos 30 para os 60 dias e decréscimo até 90 dias. Em 16,67\% não ocorreram diferenças significativas entre os teores de glicose nos vários períodos de armazenamento. Houve aumento nos teores de glicose em $8,33 \%$ dos tratamentos e decréscimo também em $8,33 \%$ dos tratamentos.

Os porcentuais de glicose variaram de $0,47 \%$ a $5,29 \%$, enquanto que as variações nos porcentuais de 


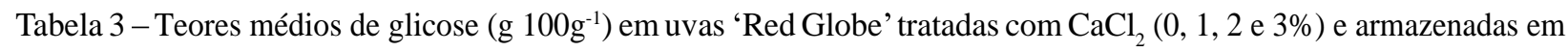
temperatura ambiente por 0,3 e 6 dias e em temperatura refrigerada por 30,60 e 90 dias.

\begin{tabular}{|c|c|c|c|c|c|c|c|c|c|}
\hline & \multicolumn{9}{|c|}{ Tempo de armazenamento em temperatura ambiente (dias) } \\
\hline & & 0 & & & 3 & & & 6 & \\
\hline \multirow{2}{*}{$\begin{array}{l}\text { Concentração } \\
\text { de } \mathrm{CaCl}_{2}(\%)\end{array}$} & \multicolumn{9}{|c|}{ Tempo de armazenamento refrigerado (dias) } \\
\hline & 30 & 60 & 90 & 30 & 60 & 90 & 30 & 60 & 90 \\
\hline $0 \%$ & $\begin{array}{l}2,33 \\
(\mathrm{aA})\end{array}$ & $\begin{array}{l}2,08 \\
(\mathrm{bA})\end{array}$ & $\begin{array}{l}2,68 \\
(\mathrm{bA})\end{array}$ & $\begin{array}{l}4,38 \\
(\mathrm{dC})\end{array}$ & $\begin{array}{l}3,68 \\
\text { (bB) }\end{array}$ & $\begin{array}{l}2,46 \\
(\mathrm{aA})\end{array}$ & $\begin{array}{l}4,71 \\
(\mathrm{dC})\end{array}$ & $\begin{array}{l}2,82 \\
(\mathrm{cA})\end{array}$ & $\begin{array}{l}3,57 \\
\text { (bB) }\end{array}$ \\
\hline $1 \%$ & $\begin{array}{l}3,87 \\
(\mathrm{cA})\end{array}$ & $\begin{array}{l}4,17 \\
(\mathrm{dA})\end{array}$ & $\begin{array}{l}4,23 \\
(\mathrm{dA})\end{array}$ & $\begin{array}{l}1,51 \\
(\mathrm{aA})\end{array}$ & $\begin{array}{l}1,85 \\
(\mathrm{aA})\end{array}$ & $\begin{array}{l}3,05 \\
(\mathrm{bB})\end{array}$ & $\begin{array}{l}1,07 \\
(\mathrm{aB})\end{array}$ & $\begin{array}{l}0,47 \\
(\mathrm{aA})\end{array}$ & $\begin{array}{l}3,71 \\
\text { (bC) }\end{array}$ \\
\hline $2 \%$ & $\begin{array}{l}3,31 \\
\text { (bB) }\end{array}$ & $\begin{array}{l}0,86 \\
(\mathrm{aA})\end{array}$ & $\begin{array}{l}3,34 \\
(\mathrm{cB})\end{array}$ & $\begin{array}{l}3,69 \\
(\mathrm{cA})\end{array}$ & $\begin{array}{l}3,59 \\
(\mathrm{bA})\end{array}$ & $\begin{array}{l}5,29 \\
(\mathrm{~dB})\end{array}$ & $\begin{array}{l}3,03 \\
(b B)\end{array}$ & $\begin{array}{l}1,63 \\
(\mathrm{bA})\end{array}$ & $\begin{array}{l}3,28 \\
\text { (bB) }\end{array}$ \\
\hline $3 \%$ & $\begin{array}{l}2,53 \\
(\mathrm{aB})\end{array}$ & $\begin{array}{l}3,17 \\
(\mathrm{cC})\end{array}$ & $\begin{array}{l}1,44 \\
(\mathrm{aA})\end{array}$ & $\begin{array}{l}2,86 \\
\text { (bB) }\end{array}$ & $\begin{array}{l}2,21 \\
(\mathrm{aA})\end{array}$ & $\begin{array}{l}4,16 \\
(\mathrm{cC})\end{array}$ & $\begin{array}{l}4,16 \\
(\mathrm{cB})\end{array}$ & $\begin{array}{l}4,19 \\
(\mathrm{~dB})\end{array}$ & $\begin{array}{l}1,75 \\
(\mathrm{aA})\end{array}$ \\
\hline
\end{tabular}

$\mathrm{CV}=$ coeficiente de variação;

Letras maiúsculas - indicam diferenças na horizontal;

Letras minúsculas - indicam diferenças na vertical.

frutose foi de $5,93 \%$ a $8,77 \%$. Os porcentuais de frutose, em relação aos açúcares redutores totais, variaram de $54,44 \%$ a $94,94 \%$, sendo superiores aos de glicose cuja variação foi de $5,06 \%$ a $45,56 \%$. Essa superioridade em porcentuais de frutose está de acordo com alguns autores (CARVALHO \& CHITARRA, 1984; CHITARRA \& CHITARRA, 2005; PEYNAUD, 1997) que afirmam que nas uvas maduras a relação frutose: glicose deve ser sempre maior que 1 (um) para que haja grau de doçura adequado. Os níveis mais elevados de frutose são vantajosos, uma vez que seu poder adoçante ao ser comparado com o do sacarose (100) é de 180, enquanto o da $\alpha$-D-glicose é de 74 e da $\alpha$-D-glicose é de 82 (FENNEMA, 1993).

$\mathrm{O}$ cálcio diminui a atividade respiratória e conseqüentemente acumula os açúcares o que justifica o aumento ocorrido nesse trabalho.

No presente trabalho, a uva 'Red Globe' apresentou no período pós-colheita alterações nos constituintes físicos, físico-químicos e químicos. Essas alterações já foram observadas por Chervin et al. (2004) ao avaliar uvas 'Carbenet Sauvignon', durante o período do veraison, usando 1-metil ciclopropeno, concluindo que os dados não levam a considerar a uva como um fruto climatérico, porém, que a utilização de novas técnicas e novas ferramentas de trabalho poderiam mudar a classificação dos frutos quanto ao amadurecimento.

\section{CONCLUSÃO}

Conclui-se que tratamentos com $\mathrm{CaCl}_{2}$ propicia às uvas melhor sabor, sendo indicada a concentração de $\mathrm{CaCl}_{2}$ $2 \%$, o armazenamento a frio por 60 dias e em temperatura ambiente até 6 dias.

\section{REFERÊNCIAS BIBLIOGRÁFICAS}

ASSOCIATION OF OFFICIAL ANALYTICAL CHEMISTS. Official methods of analysis of Associacion of Official Analytical Chemists. Virginia, 1990. $1094 \mathrm{p}$.

BRADY, C. Y. Fruit ripenig. Annual Review of Plant Physiology, Palo Alto, v. 38, p. 155-178, 1987.

CAMPOS, A. J. de; MANOEL, L.; VIEITES, R. L. Utilização da irradiação na qualidade pós colheita de uva 'Itália'. In: CONGRESSO BRASILEIRO DE FRUTICULTURA: TECNOLOGIA, COMPETITIVIDADE E SUSTENTABILIDADE, 18., 2004, Florianópolis. Anais... Florianópolis: SBF, 2004. p. 1-4.

CARVALHO, V. D. de; CHITARRA, M. I. F. Aspectos qualitativos da uva. Informe Agropecuário, Belo Horizonte, v. 10, n. 117, p. 75-79, set. 1984. 
CENCI, A. S. Ácido naftaleno acético (ANA) e cloreto de cálcio na pré-colheita de uva 'Niagara Rosada' (Vitis labrusca $\mathbf{x}$ Vitis vinifera $\mathrm{L}$ ): avaliação do potencial de conservação no armazenamento. 1994. 109 f. Tese (Doutorado em Ciências dos Alimentos) - Universidade Federal de Lavras, Lavras, 1994.

CHERVIN, C.; El-KEREAMY, A.; ROUSTAN, J. P.; LATCHÉ, A.; LAMDN J.; BOUZAYEN. Ethylene seems required for the berry development and ripening in grape, a nonclimateric fruit. Plant Science, Oxford, v. 167, n. 6, p. 13011305,2004

CHITARRA, M. I. F.; CHITARRA, A. B. Pós-colheita de frutos e hortaliças: fisiologia e manuseio. 2. ed. rev. e ampl. Lavras: UFLA, 2005. 785 p.

FENNEMA, R. O. Química de los alimentos. Zaragoza: Acribia, 1993. 1095 p.

FERREIRA, D. F. Análises estatísticas por meio do SISVAR para windows versão 4.0. In: REUNIÃO ANUAL DA REGIÃO BRASILEIRA DA SOCIEDADE INTERNACIONAL DE BIOLETRIA, 45., 2000, São Carlos. Resumos... São Carlos: UFSCar, 2000. p. 235.

FRÁGUAS, J. C.; SILVA, D. J. Nutrição e adubação da videira em regiões tropicais. Informe Agropecuário, Belo Horizonte, v. 19, n. 194, p. 70-75, 1998.
KADER, A. A. Fruit maturity ripening on quality relationships. Acta Horticulture, Leuven, n. 485, p. 203208, 1999.

NELSON, N. A. A. Photometric adaptation of Somogy method for the determination of glucose. Journal of Biological Chemistry, Baltimore, v. 135, n. 1, p. 136-175, Jan. 1994.

NOGUEIRA, D. J. P. Clima na viticultura. Informe Agropecuário, Belo Horizonte, v. 10, n. 117, p. 11-14, set. 1984.

PEREIRA, G. E. Caracterização agronômica de cultivares de videira para suco em Minas Gerais: avaliação analítica e sensorial dos sucos. 2001. 126 p. Dissertação (Mestrado em Ciência dos Alimentos) - Universidade Federal de Lavras, Lavras, 2001.

PEYNAUD, E. Connaissance et travel du vin. 2. ed. Paris: Dunod, 1997. $341 \mathrm{p}$.

RIBÉREAU-GAYON, P.; REYNAUD, E. Traitéd'oenologie. 2. ed. Paris: Beranger, 1964.

RIZZON, L. A.; MANFROI, V.; MENEGUZZO, J. Elaboração de suco de uva na propriedade vitícola Bento Gonçalves. Bento Gonçalves: Embrapa-CNPUV, 1998. 24 p.

WANG, C. Y. Postharvest quality decline, quality marten ace e quality evaluation. Acta Horticulture, Leuven, v. 485, p. 389-392, Aug. 1999. 